Research Article

\title{
The Gut Microbiome Modulates the Changes in Liver Metabolism and in Inflammatory Processes in the Brain of Chronic Unpredictable Mild Stress Rats
}

\author{
Wei-jie Lv $\mathbb{D},{ }^{1}$ Xiao-ling Wu $\mathbb{D},{ }^{2}$ Wen-qian Chen $\mathbb{D}^{1},{ }^{1}$ Yue-fei Li $\mathbb{D},{ }^{1}$ Gui-feng Zhang $\mathbb{D}{ }^{2}$ \\ Li-min Chao $\odot,{ }^{1}$ Jia-hao Zhou $₫$, ${ }^{1}$ Ao Guo $\odot,{ }^{1}$ Cui Liu $\odot{ }^{1,3,4,5}$ and Shi-ning Guo ${ }^{1,3,4,5}$ \\ ${ }^{1}$ College of Veterinary Medicine, South China Agricultural University, Guangzhou, Guangdong 510642, China \\ ${ }^{2}$ Zhaoqing Medical College, Zhaoqing, Guangdong 526020, China \\ ${ }^{3}$ Guangdong Research Center for Veterinary Traditional Chinese Medicine and Natural Medicine Engineering Technology, \\ Guangzhou, Guangdong 510642, China \\ ${ }^{4}$ Guangdong Provincial Key Laboratory of Prevention and Control for Severe Clinical Animal Diseases, Guangzhou, \\ Guangdong 510642, China \\ ${ }^{5}$ International Institute of Traditional Chinese Veterinary Medicine, Guangzhou, Guangdong 510642, China
}

Correspondence should be addressed to Cui Liu; liuc@scau.edu.cn and Shi-ning Guo; shining@scau.edu.cn

Received 5 June 2019; Revised 27 August 2019; Accepted 7 September 2019; Published 24 October 2019

Guest Editor: Fabrizio Biundo

Copyright (c) 2019 Wei-jie Lv et al. This is an open access article distributed under the Creative Commons Attribution License, which permits unrestricted use, distribution, and reproduction in any medium, provided the original work is properly cited.

\begin{abstract}
Generally, depression is the result of complex gene-environment interactions. Recent studies have showed that the gut microbiota can affect brain function through the microbiota-gut-brain axis. However, the underlying mechanism of the microbiota and potential influence of depression remain elusive. We aimed to determine how gut microbiome contributes to the process of depression and further influences the host. Chronic unpredictable mild stress (CUMS) is used to establish a depression model. Fecal microbiota transplant (FMT) is applied to illustrate that depression can be transmitted via microbiota, and metabolism of liver analysis is applied to demonstrate further influence to the liver. We also analyzed the astrocyte activation in the brain by immunofluorescence (IF). Here, we show that the structure of the gut microbiome changes markedly after rats undergo CUMS. Notably, we found that the ratio of Lactobacillus to Clostridium can be a vital index for the development of depression. Depression-like behavior can be duplicated through FMT. Moreover, increased zonulin and fatty acid binding protein-2 indicates that gut barrier integrity is broken after FMT. Subsequently, metabolomics shows that liver metabolic disorder occurs and leads to liver coagulative necrosis. In addition, increased inflammatory cytokine expression and higher astrocyte activation indicate an inflammatory process in the brain. These findings suggest that dysbiosis gut microbiome contributes to development of depression and further causes liver metabolic disorders in a way that may be relevant to the Lactobacillus to Clostridium ratio.
\end{abstract}

\section{Introduction}

Depression is a common mood disorder that can lead to suicide attempts [1] and accounting for $12.3 \%$ of the global burden of disease rising to $15 \%$ by 2020 [2]. Although, the cause of depression is unclear, many aspects of the biological, psychological, and social environments are certainly involved in the pathogenesis of depression. These biological factors mainly involve genetics, neurobiochemistry, neuroendocrine function, and nerve regeneration $[3,4]$.
Evidence from animal models and clinical studies suggests that the gut microbiome may play a crucial role in the central nervous system function $[5,6]$. The mechanism by which the gut microbiome and the brain interact is not fully understood; it may be related to the stress-induced leaky gut [7]. These previous studies highlight the potential link between changes in the gut microbiome structure and the development of depression, and the microbe-gut-brain axis has been proposed to elucidate the relationship between the gut microbiome and the brain [8]. 
Studies have paid much attention to changes in the brain and neurons, but it is unclear how gut microbiome contributes to the development of depression and what the further influence is to the host. Zheng et al. have shown that the development of depression is related to metabolic disorders [9]. Interestingly, some of the metabolites that are significantly altered in patients with depression (such as hippuric acid, dimethylamine, and dimethylglycine) are metabolites of the gut microbiome [9]. The liver receives blood from the intestines, metabolizes the products of the gut microbiome, and regulates bile acid signaling [10]. As a hormone, bile acid, combined with other microbial molecules to control metabolism, strongly shapes liver metabolism [10]. In addition, intestinal microbial metabolites can be sensed by liver immune cells, which can disrupt liver homeostasis, leading to fibrosis and liver cancer [10]. A recent clinical study and a rodent model research have shown that compared with the control group, the expression of lipopolysaccharide (LPS) biosynthesis-related genes was increased in patients with anxiety and depression $[11,12]$. The underlying mechanism may be due to the disruption of the gut barrier induced by changed gut microbiome and various toxins entering the blood, resulting in a series of neuropsychiatric symptoms. Meanwhile, the reduction of serotonin released by intestinal enterochromaffin cells (EC) promotes the development of depression [13]. Based on these findings, we hypothesize that the ecological imbalance of the gut microbiome may break the gut barrier integrity and inhibit EC to express 5-HT, subsequently contributing to the liver metabolic disorders and development of brain inflammation.

In this study, we establish a CUMS model in rats, use the behavioral test to assess whether the rats exhibit depression-like behaviors, and apply $16 \mathrm{~S}$ rRNA gene sequencing technology to identify changes in the gut microbiome. In addition, to demonstrate whether depression can be transmitted through the gut microbiome, we transplant feces from CUMS rats into antibiotic-treated rats. Moreover, metabolomics analysis and the enzyme-linked immunosorbent assay (ELISA) test are performed to determine how the gut microbiome affects host liver metabolism and brain inflammation.

\section{Results}

2.1. Evaluation of the Effect of CUMS. To test whether CUMS affects rat behavior, we performed different behavioral tasks at the end of the study. We examined four parameters to evaluate the CUMS rat model, specifically body weight (BW) and performance on the sucrose preference test (SPT), the open field test (OFT), and the tail suspension test (TST). There were significant differences between the control group and the model group. Compared with the control group $(371.14 \pm 10.08 \mathrm{~g})$, the model group $(285.77 \pm 14.75 \mathrm{~g})$ exhibited significantly decreased body weight $(p<0.01)$ (Figure 1(a)).

Sucrose preference is used to test whether a rat exhibits a deficit in happiness, which is an important indicator of depression [14]. Our results indicate that the model group exhibits reduced sucrose preference. There was no significant difference at day 0 , but the sucrose preference of the model group $(62.50 \pm 12.61 \%)$ was significantly lower compared with that of the control group $(82.83 \pm 6.11 \%)(p<0.01)$ (Figure 1(b)).

When rats are in an open field, they tend to exhibit reduced activity in the central area, which is related to depression-like behavior [15]. Therefore, we used the open field test to determine whether CUMS causes depressionlike behaviors, and the time spent in the central region was used as an indicator of depression-like behaviors. No significant difference was observed at day 0 , but the rats in the model group $(0.51 \pm 1.05 \mathrm{~s})$, which underwent CUMS for 28 days, showed a significant reduction in the time spent in the central region compared with that exhibited by the rats in the control group $(8.90 \pm 5.04 s)(p<0.01)$ (Figure $1(c))$.

Finally, the tail suspension test, originally developed by Steru and colleagues [16], is very effective in animal models of depression and can be used as a test to predict depression-like behaviors. In this study, we used rat immobility time as an indicator of depression phenotype. Compared with that of the control group $(33.20 \pm 7.84 \mathrm{~s})$, the immobility time of the model group $(154.16 \pm 26.08 \mathrm{~s})$ increased markedly $(p<0.01)$ (Figure $1(d)$ ). From the open field test, the motion tracks of a control rat and a model rat in the open field test are shown in Figures 1(e) and 1(f). These findings showed that rats express a depression-like behavior after undergoing CUMS.

\subsection{Alterations in the Microbiome after CUMS}

2.2.1. $\alpha$ Diversity. Previous studies have reported that CUMS-induced depression can be alleviated via the alteration of microbiome $[17,18]$. In our study, $\alpha$ diversity, as measured by the Chaol and Shannon Indices, was reduced after CUMS (Figures 2(a)-2(d)); the Shannon Index especially is significantly decreased compared with that of the control group, which means that the diversity of OTU changed markedly, whereas the richness of OTU had a slight decrease after CUMS.

2.2.2. $\beta$ Diversity. In general, the distance between the same groups of samples reflects the difference between the individuals within the group. If the difference between the groups is significantly higher than the differences within one group, it indicates that there is a significant difference between the two groups. As shown in Fig. S1, all of the distances between the groups are higher than all of the distances within the groups. To test the similarity between the two groups, an unweighted pair-group method with arithmetic means was performed (Fig. S1). Meanwhile, principal component analysis (PCA), principal coordinates analysis (PCoA), and partial least squares discrimination analysis (PLS-DA) were performed. As shown in Figures 2(e) and 2(f) (Fig. S1), the model group was significantly separated from the control group after CUMS. These results suggest that CUMSinduced depression can change the structure of microbiome.

2.3. Taxonomy Analysis. To identify the significant changes in the gut microbiome between the model group and the control group, we used QIIME software to obtain the 

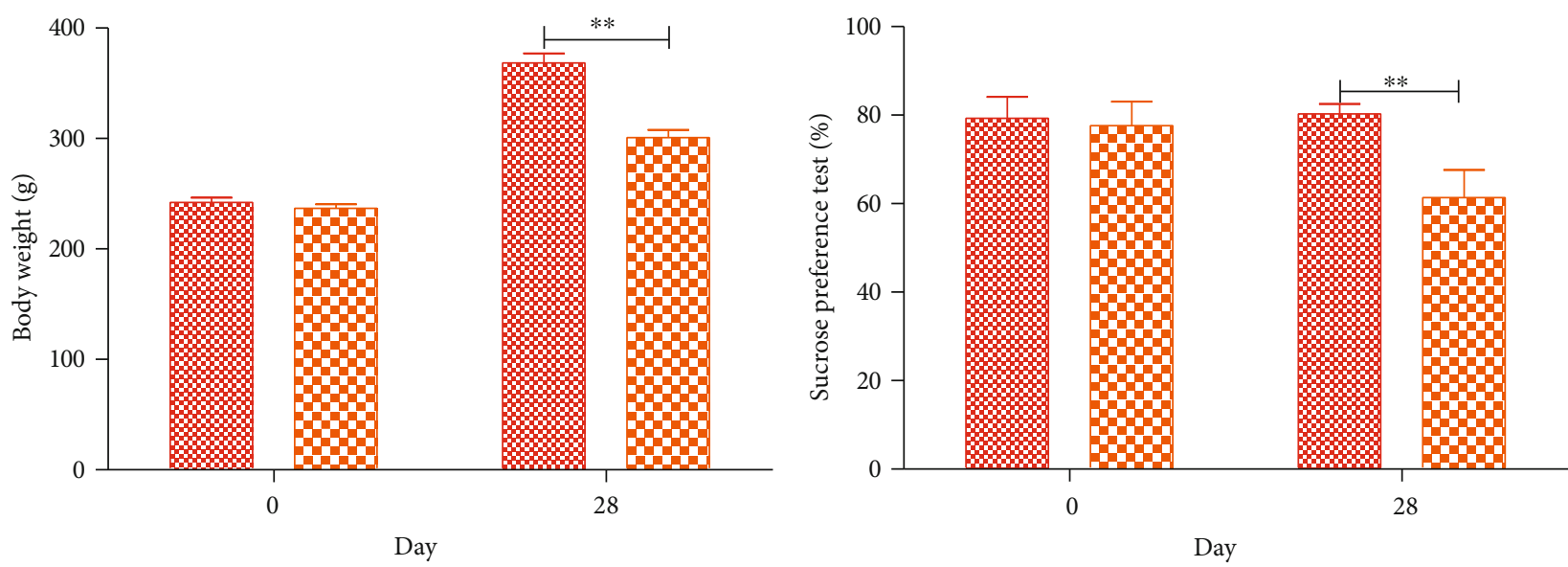

\%on

DII Mod

(a)

(b)
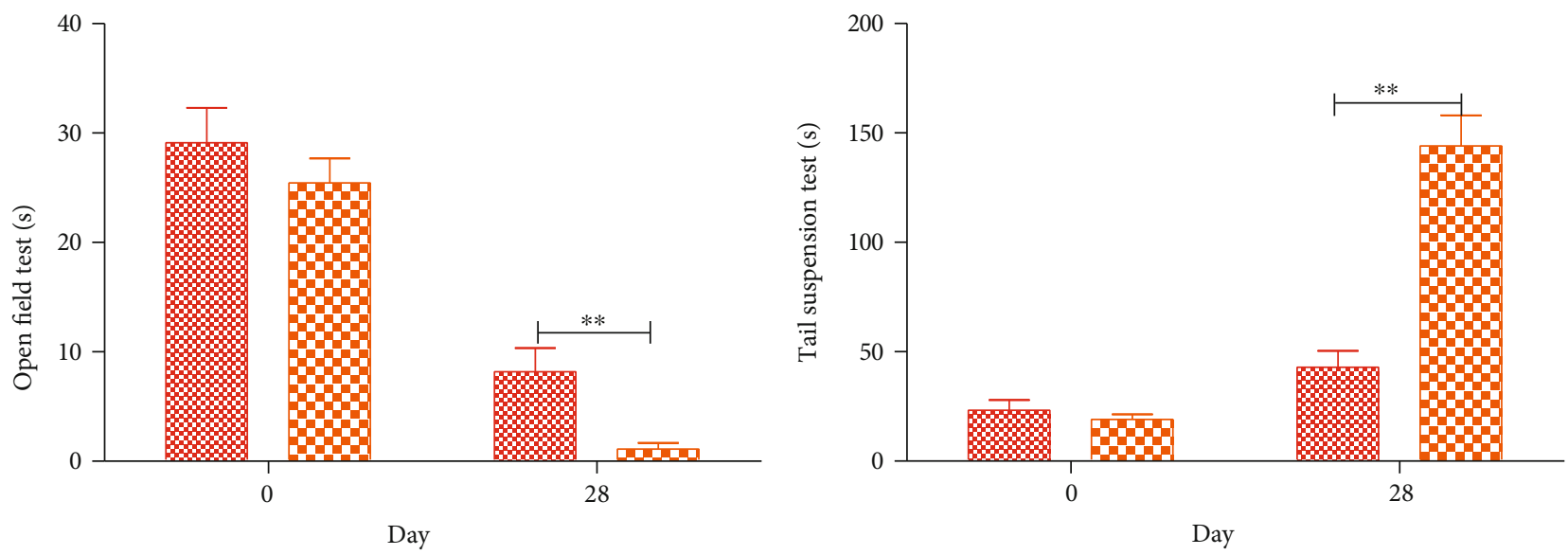

Fon

표 Mod

(c)

Kon

DII Mod

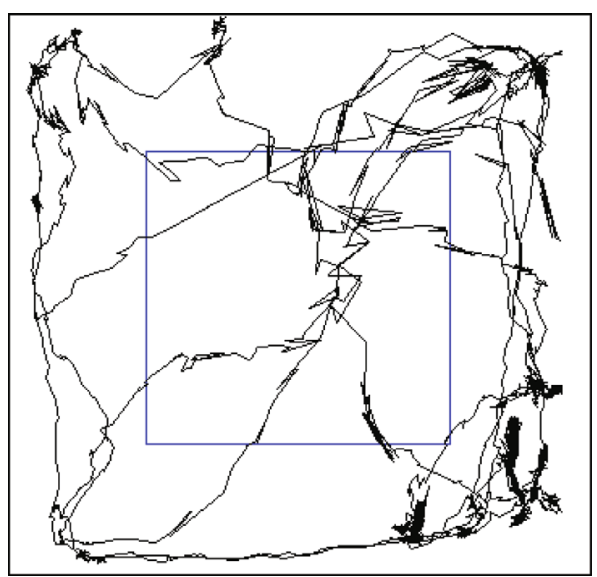

(e)

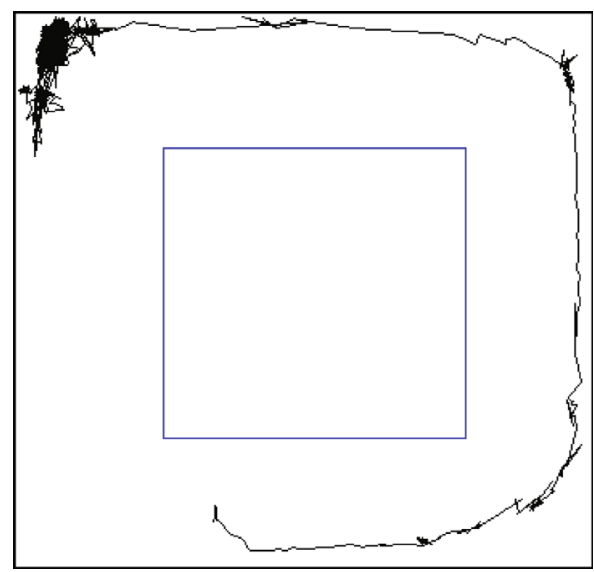

(f)

FIGURE 1: Behavioral test: (a) change in body weight in both groups during the CUMS period; (b) results of the sucrose preference test (SPT); (c) results of the open field test (OFT); (d) results of the tail suspension test (TST); (e, f) representative motion tracks for a control rat and a model rat. All data are represented by the mean \pm SEM $(n=6)$ by $t$-test, ${ }^{* *} p<0.01$. 


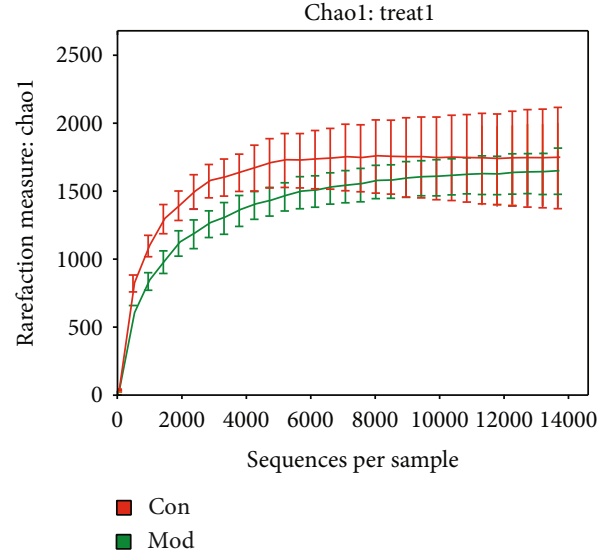

(a)

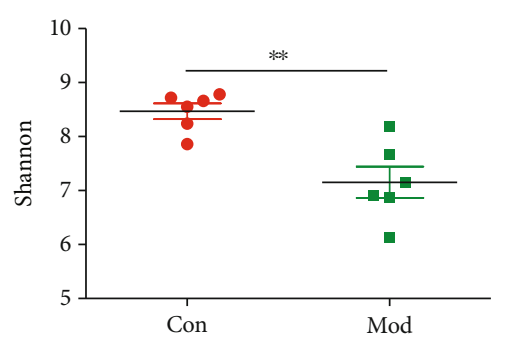

(d)

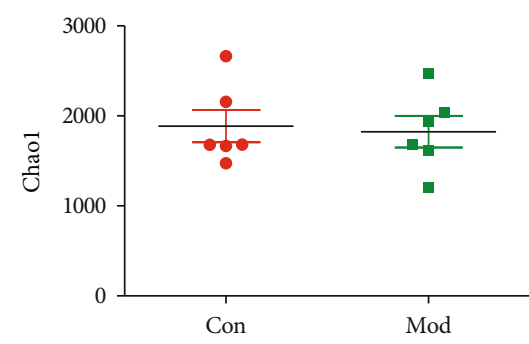

(b)

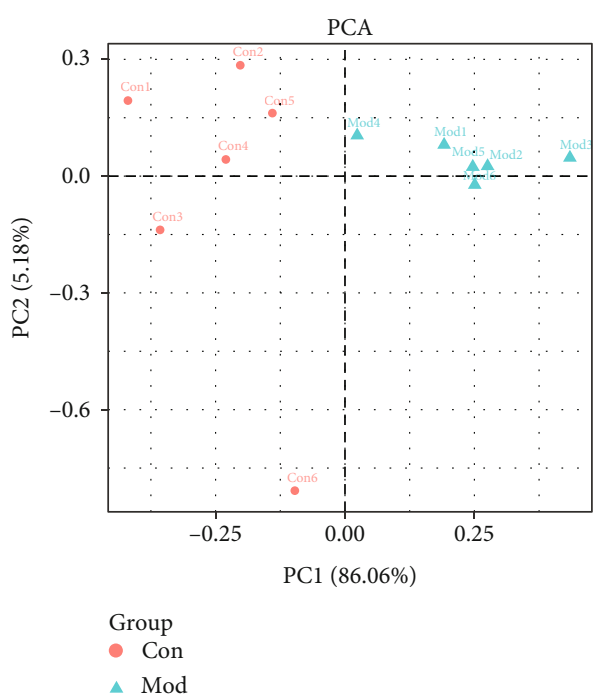

(e)

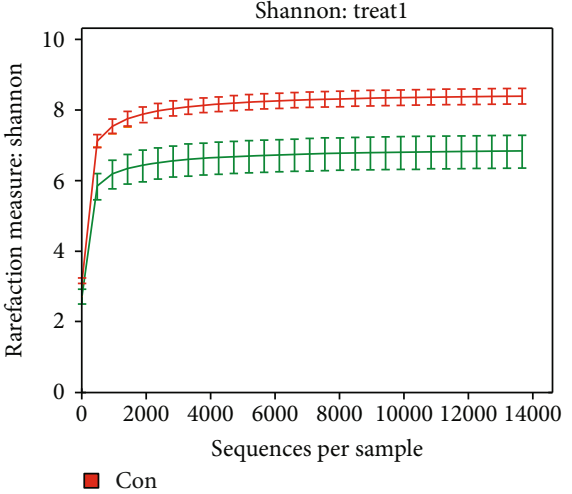

(c)

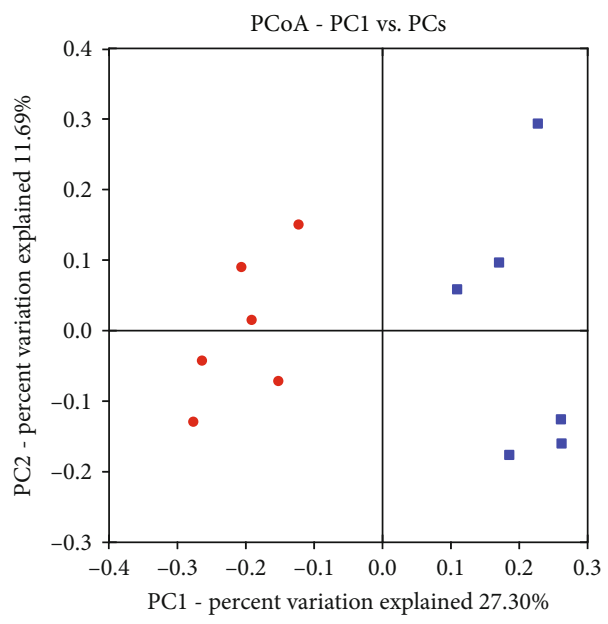

Group

- Con

- Mod

(f)

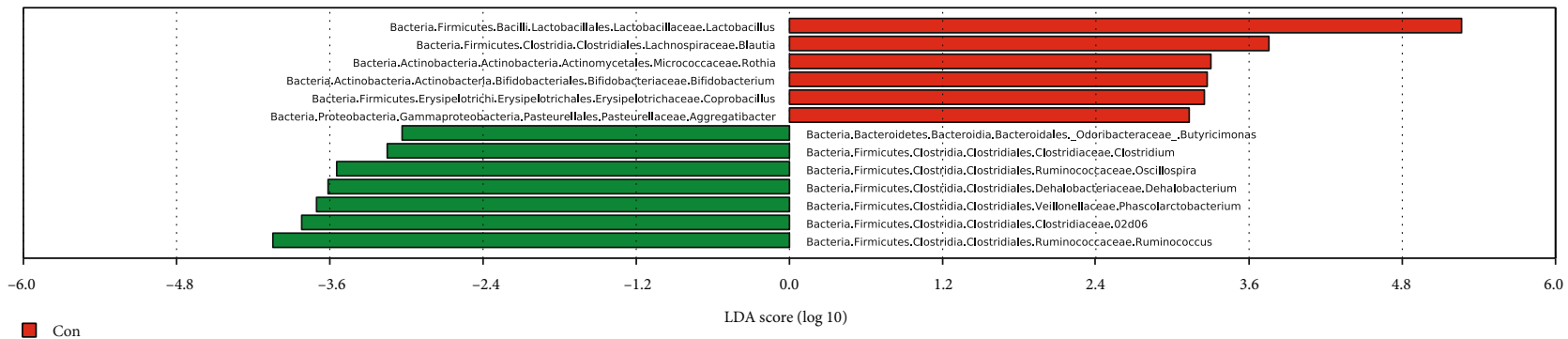

(g)

Figure 2: Continued. 


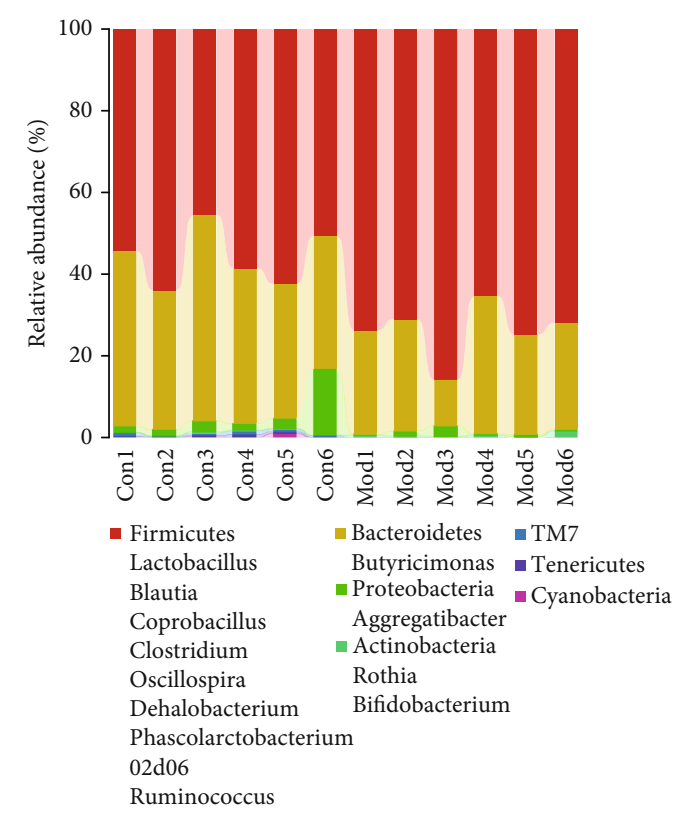

(h)
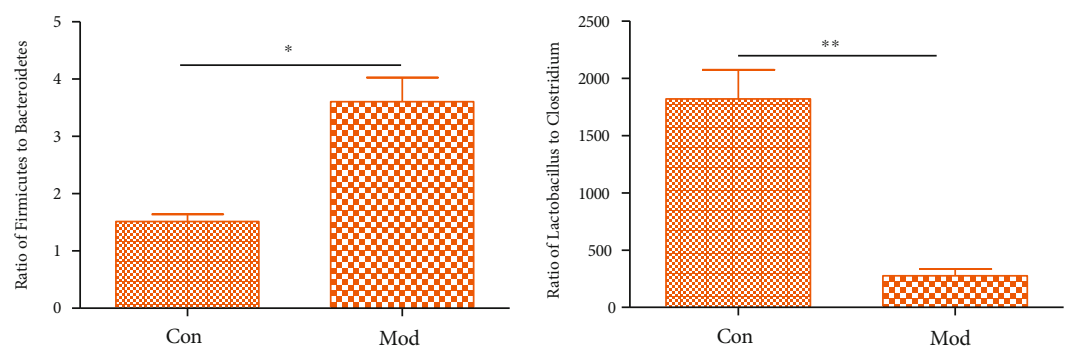

(i) (j)

FIGURE 2: Alterations of microbiota after CUMS: (a) Chaol Index; (b) analyses of Chaol; (c) Shannon Index; (d) analyses of Shannon; (e) PCA; (f) unweighted PCoA; (g) LEfSe; (h) taxonomy summary; (i) ratio of Firmicutes to Bacteroides; (j) ratio of Lactobacillus to Clostridium. Above data were presented as means \pm SEM $(n=6)$ by $t$-test. ${ }^{*} p<0.05 ;{ }^{* *} p<0.01$.

composition and abundance distribution table of each sample at the five classification levels (the phyla, the class, the order, the family, and the genera), and the results of the analysis were presented in a histogram. After rats underwent CUMS, the structure of the microbiome was significantly changed. Six and seventeen species were most distinct at the phylum and genus levels, respectively (Fig. S2). Using Mothur Software, we tested the difference in sequence between the two groups at the phylum and genus levels. The classification tree shows the hierarchical relationship of all taxon units in the community from phylum to genus. A total of 13 genera were identified, and their relative abundance was able to distinguish the model group from the control group (Figure 2(g)). Among the 13 genera, 6 genera were higher in the model group; these genera belong to the following classes: Bacilli, Clostridia, Actinobacteria, Erysipelotrichi, and Gammaproteobacteria. Meanwhile, 7 genera were higher in the control group; these genera belong to the following classes: Bacteroidia and Clostridia. The discriminative genera mainly belonged to the phyla Firmicutes (9/13, $69.23 \%)$, Actinobacteria $(2 / 13,15.38 \%)$, Bacteroidetes (1/13, $7.69 \%$ ), and Proteobacteria (1/13, 7.69\%) (Figure 2(h)). Compared with the control group, the model group exhibited increased relative abundances of Actinobacteria and Firmicutes and a decreased relative abundance of Bacteroidetes (Fig. S2). A lower ratio of Firmicutes to Bacteroidetes (F/B) is considered to be a key index for a healthy state of the gut microbiome $[19,20]$. In our study, the ratio of $\mathrm{F} / \mathrm{B}$ was increased in the model group $(p=0.045)$ (Figure 2(i)). Lactobacillus rhamnosus, Lactobacillus acidophilus, and Lactobacillus reuteri, belonged to Lactobacillus, have been considered to have anti-inflammatory function [21, 22], whereas higher Clostridium abundance usually is associated with gut inflammation [23-25]. So, we measured the ratio of Lactobacillus to Clostridium (L/C). Here, the ratio of L/C was decreased $(p=0.004)$ (Figure $2(j))$ in the model group relative to the control group. These results suggest that the ratio of L/C may be a potential index of depression, and the decreased ratio of $\mathrm{L} / \mathrm{C}$ may indicate promotion of the process of depression.

2.4. Transplantation of Model Rat Microbiome Induces Depression-Like Behaviors in Antibiotic-Treated Rats. To verify the causal relationship between the gut microbiome and depression, we performed a fecal microbiome transplant experiment. This method has an important role for determining the pathogenicity of intestinal microbiome in obesity, colitis, and type I diabetes [26-28]. Here, as previously reported, feces derived from the model group or from the control group were transplanted to antibiotic-treated rats.

The body weight of the rats was measured daily. No significant difference in body weight was observed between the FMT group and the control group during the FMT experiment (Figure 3(a)). As previous research [29], tests for depression-like behaviors (the SPT, OFT, and TST) were performed on day 7 after FMT to test whether depression-like behaviors were present in rats after FMT. On the 7th day after FMT, there was a significant difference in behavior in the OFT and TST between the control group and FMT group rats. However, a significant difference in behavior in the SPT between the FMT group and the control group was not observed (Figure 3(b)). Compared with the control group, the FMT group rats showed a decrease in the proportion of time spent in the central area in the OFT and an increase in the duration of immobility in the TST (Figures 3(c) and $3(d)$ ). These results suggest that depression-like behaviors 

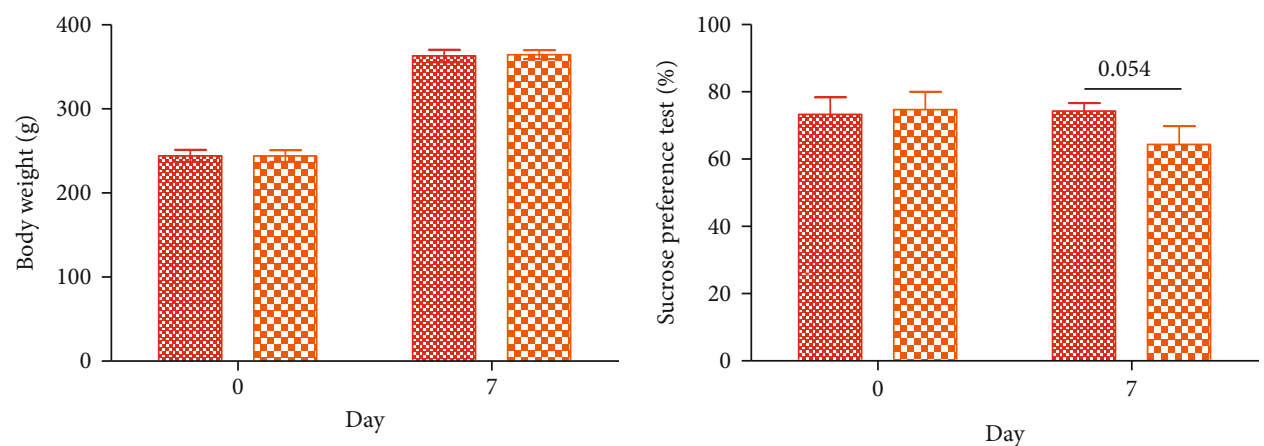

ॠ Con BD FMT

(a)

Con

FMT

(b)
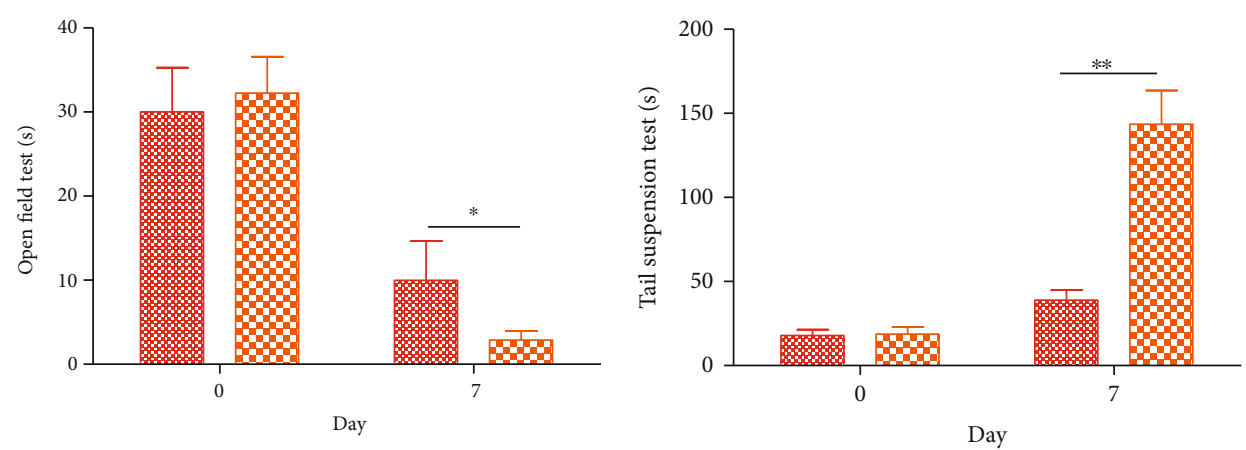

Fi맘 FMT

@ Con

묘요 FMT

(c)

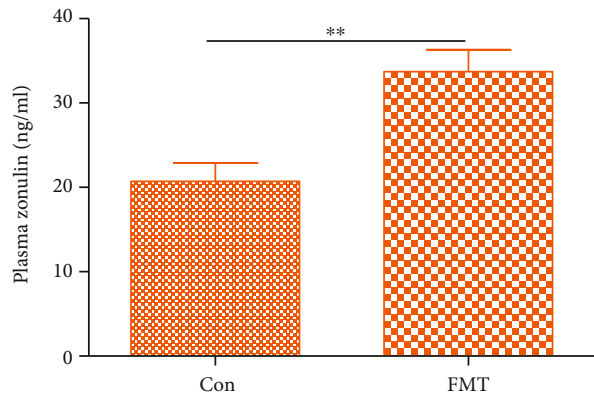

(e)

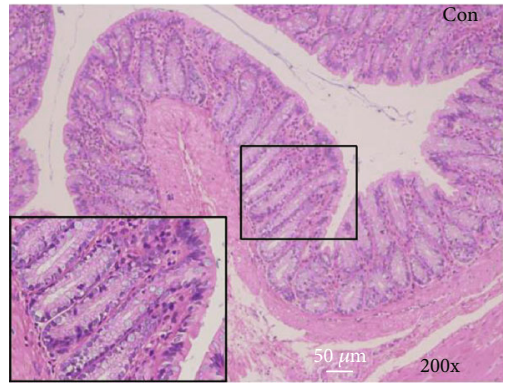

(g)

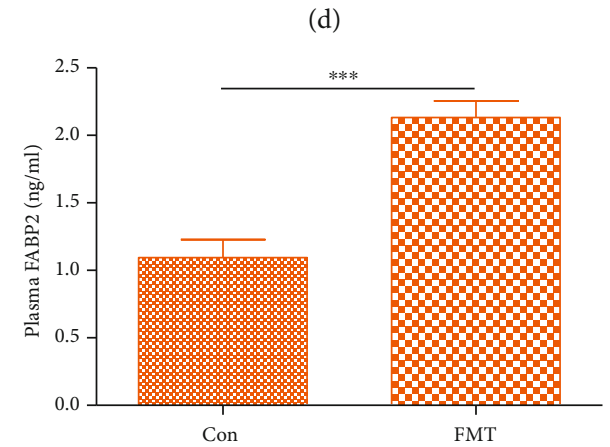

(f)

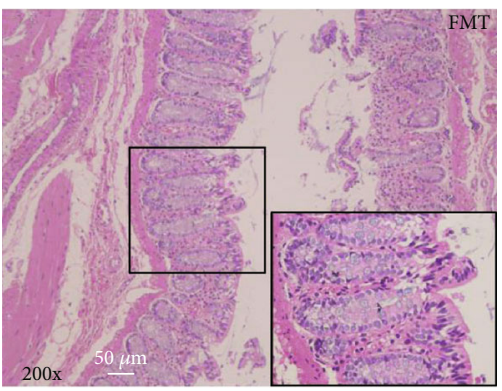

(h)

Figure 3: Continued. 


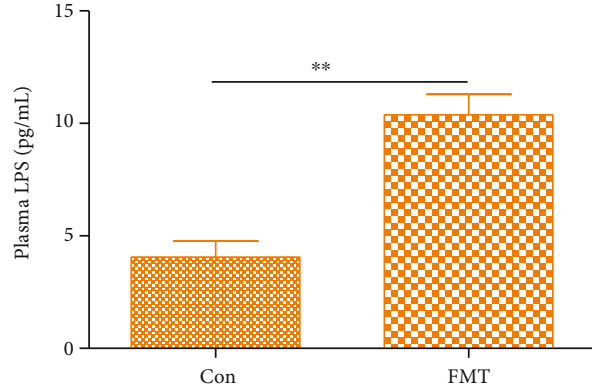

(i)

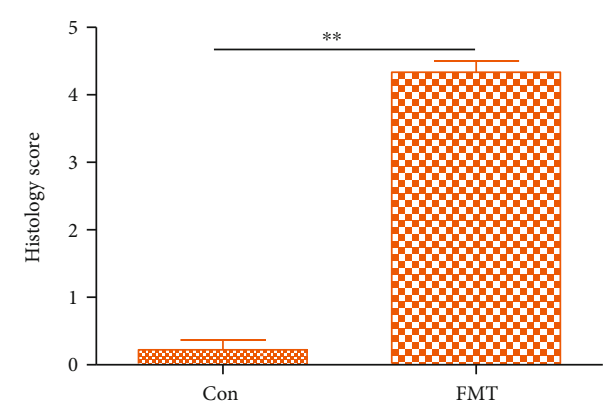

(j)

FIGURE 3: Behavioral test after FMT: (a) body weight; (b) results of the sucrose preference test (SPT); (c) results of the open field test (OFT); (d) results of the tail suspension test (TST); (e) expression of zonulin; (f) expression of FABP2; (g) the colon in the control group presents the normal histological feature; (h) epithelial cells are necrotic and shedding after FMT; (i) LPS in plasma; (j) colon histology score. Scale bar: $50 \mu \mathrm{m}$. All data are represented by the mean \pm SEM $(n=6)$ by $t$-test; ${ }^{*} p<0.05,{ }^{* *} p<0.01$, and ${ }^{* * *} p<0.001$.

in the OFT, FST, and TST can be transmitted through the gut microbiome. In addition, previous research has demonstrated that increased human intestinal barrier permeability plasma biomarkers zonulin and fatty acid binding protein-2 (FABP2) correlated with plasma lipopolysaccharide (LPS) and altered gut microbiome in anxiety and depression [11]. Here, LPS, zonulin, and FABP2 expressions were higher than the control group in plasma in the FMT group (Figures 3(e), $3(\mathrm{f})$, and 3(i)), indicating that the intestinal barrier has been broken after FMT. Additionally, the colon histological changes showed that epithelial cells are necrotic and shedding after FMT (Figures 3(g), 3(h), and 3(j)). These findings indicate that depression can be transmitted via FMT; furthermore, depression-like microbiome induces intestinal barrier disruption.

2.5. Alteration of Liver Metabolites in FMT Rats. OPLS-DA was performed on liver metabolites in the FMT group and the control group. The OPLS-DA score map shows a clear separation between FMT and control rats using liquid chromatograph mass spectrometer (LC-MS) metabolomics (LCMS pos: $\mathrm{R} 2 \mathrm{Y}=0.997, \mathrm{Q} 2=0.824$; LC-MS neg: $\mathrm{R} 2 \mathrm{Y}=0.973$, $\mathrm{Q} 2=0.633$ ) (Fig. S3). OPLS-DA identified 74 metabolites that were significantly different between FMT rats and control rats. The details of the metabolites are shown in Table S1 and Fig. S4.

Liver metabolites were analyzed by the MetaboAnalyst and KEGG databases to investigate whether the depression-like microbiome causes liver metabolic disorders. Here, 36 pathways that were different in FMT rats compared with control rats with a $p<0.05$ were identified (Table S2). When the false discovery rate (FDR) was used to adjust the $p$ values, only 24 pathways were found to be significantly changed, and 12 of these pathways were assigned to the category "Metabolism," which included the categories "Amino acid metabolism" (3 pathways) and "Lipid metabolism" (3 pathways) (Figures 4(a) and 4(b)). Meanwhile, the levels of glutamate, glutamine, aspartate, serine, methionine, valine, leucine, isoleucine, lysine, histidine, phenylalanine, tyrosine, and tryptophan, which are involved in aminoacyl-tRNA biosynthesis [30], were significantly changed. The details of the KEGG pathways are shown in Fig. S5. Through a comprehensive analysis of liver metabolites reported in a previous study [30], glycerophospholipid metabolism was found to be disturbed in the model rats. Phosphatidylcholine (PC), a type of glycerophospholipid, not only protects cells from oxidative stress but also acts as a regulator of inflammation [31]. Additionally, the liver histological changes showed that coagulative necrosis can be observed after FMT (Figures 4(c)-4(e)). Metabolic disorders of glycerophospholipids indicate oxidative stress in the liver after FMT and are accompanied by inflammatory cell membrane damage and even apoptosis.

2.6. Inflammatory Activation Caused by FMT in the Brain. Given the evidence that depression can be related to brain inflammation [32], here, we measured the activities of brain microglia, astrocytes, and inflammatory cytokines. In this research, immunofluorescence was used to analyze microglia and astrocytes. The morphological analysis of glial fibrillary acidic protein- (GFAP/Iba-1-) positive cells revealed that 7 days of FMT exposure caused an increase in the number of activated microglia and astrocyte cells in the hippocampus of FMT rats compared to control rats, which is consistent with the criteria previously described [33] (Figures 5(a)-5(h)). The expression of the proinflammatory cytokine IL-1 $\beta$ (Figure 5(i)) and anti-inflammatory cytokine IL-10 (Figure 5(j)) was significantly increased in the FMT group. Previous research has demonstrated that the pathogenesis of depression is mainly due to a lack of 5-HT in the synaptic cleft [34]. Consistent with previous research, 5 -HT was decreased $(p=0.083)$ in FMT rats compared to the control group (Fig. S6). These results indicate that depression-like microbiome induces inflammation in the brain and may contribute to the process of depression.

\section{Discussion}

Patients with depression experience mental debilitation, and depression can even lead to suicide, which imposes a heavy burden on the social economy; however, we still do not know the pathogenesis of depression. Here, we demonstrated that 


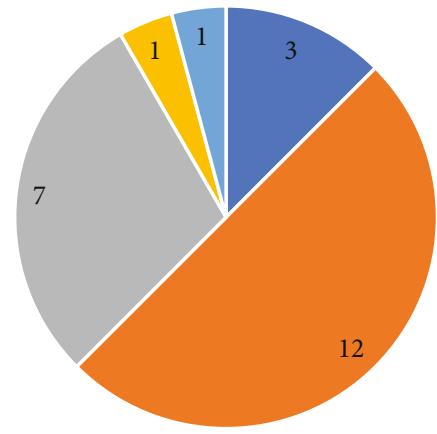

Human diseases

Metabolism

Organismal systems

Genetic information processing

Environmental information processing

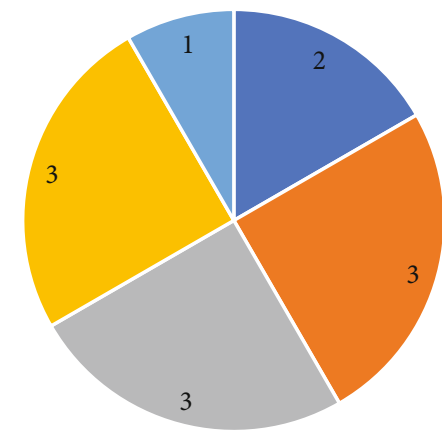

Nucleotide metabolism

Amino acid metabolism

Lipid metabolism

Metabolism of other amino acids

Carbohydrate metabolism

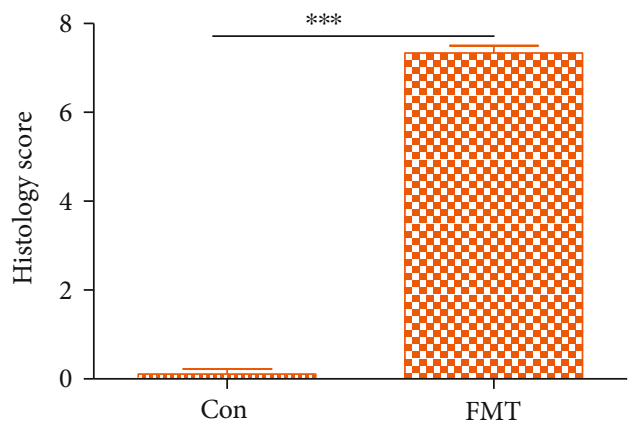

(a)

(b)

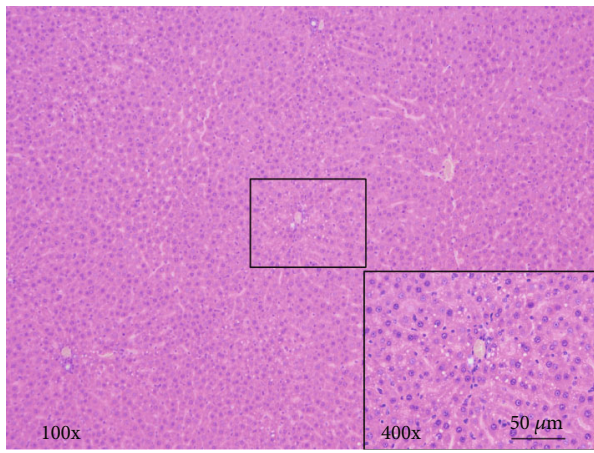

(d)

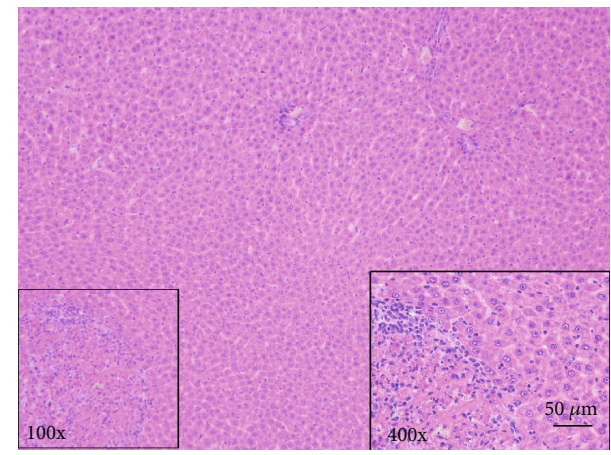

(e)

Figure 4: Changes of liver metabolism. (a) 24 pathways were significantly changed, and 12 pathways were assigned to "Metabolism." (b) "Amino acid metabolism" (3 pathways), "Lipid metabolism" (3 pathways), "Carbohydrate metabolism" (2 pathways), "Metabolism of other amino acids" (3 pathways), and "Nucleotide metabolism" (1 pathway) were assigned to "Metabolism." (c) Histology score. (d) The liver in the control group presents the normal histological feature. (e) Coagulative necrosis can be observed after FMT. Scale bar: $50 \mu \mathrm{m}$. All data are represented by the mean $\pm \operatorname{SEM}(n=6)$ by $t$-test, ${ }^{* * *} p<0.001$.

CUMS induce depression-like behavior in rats and that the structure of the intestinal microbiome in the model group is markedly altered compared to that in the control group. Interestingly, we found that the ratio of Lactobacillus to Clostridium is a potential index for the process of depression. Antibiotic-treated rats experienced depression-like behaviors after receiving a transplantation of feces from the model group, indicating that the depressed microbiome is transmissible. FABP2 and zonulin were increased after FMT, which are the major regulators of tight junctions between the endothelium and the epithelium, regulating the intestinal and blood-brain barriers [11]. Using LC-MS, 74 liver metabolites that are differentially expressed between the FMT group and the control group were identified. The metabolites that were identified included lipids, lipid metabolism-related molecules, amino acids, and other metabolites. Moreover, brain glia-astrocyte was activated, and inflammatory cytokines were increased whereas 5-HT was decreased after FMT. These results suggest that the gut microbiome contributed to host liver metabolism and brain inflammation, becoming a potential cause of depression.
Studies have shown that some species in Bacteroides are strongly associated with depression, while other species are inversely associated with depression [5]. Compared with healthy individuals, depressed patients exhibit increased levels of Bacteroidetes, Proteobacteria, and Actinobacteria and decreased levels of Firmicutes [35]. In the model group, the relative abundance of Actinobacteria and Firmicutes was higher than that in the control group, and the relative abundance of Bacteroides was lower than that in the control group. The characteristic gut microbiome of the model group in this study is not completely consistent with that found in previous studies. This difference may be due to differences in sample size or species, as well as differences in the statistical methods used to analyze the gut microbiome. However, these findings consistently indicate that the CUMS model is associated with significant changes in the structure of the gut microbiome, especially the relative abundance of Lactobacillus and Clostridium. Some of bacterial species belonging to Lactobacillus, such as Lactobacillus rhamnosus, Lactobacillus acidophilus, and Lactobacillus reuteri, have anti-inflammatory function 


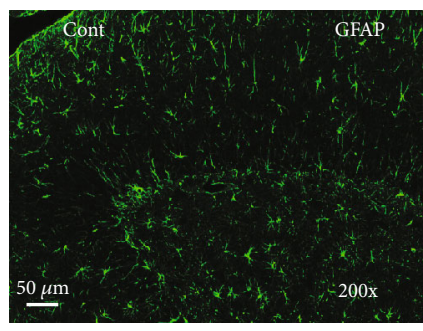

(a)

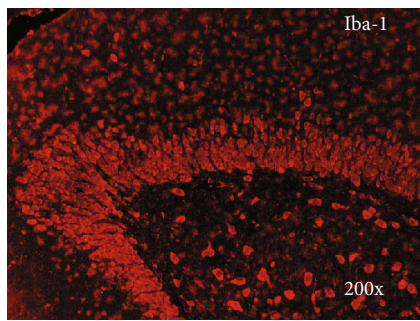

(c)

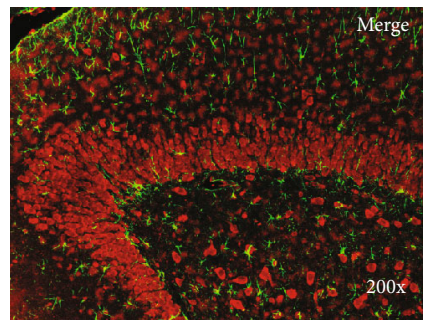

(e)

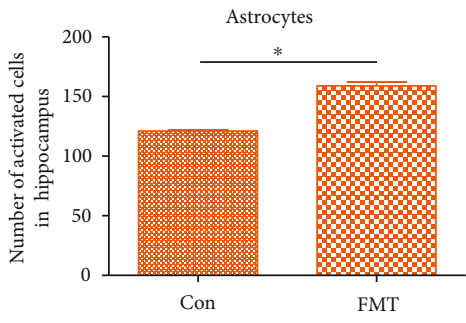

(g)

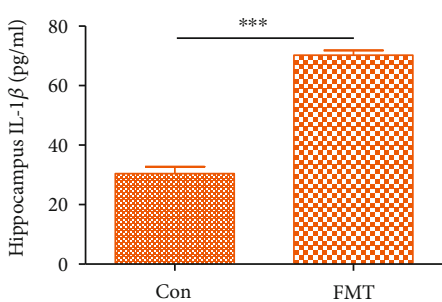

(i)

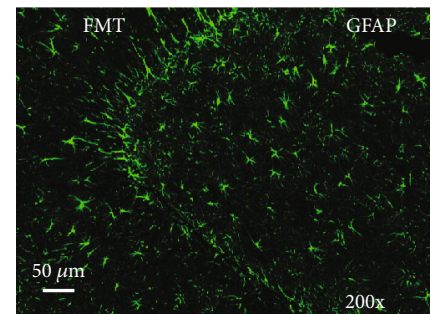

(b)

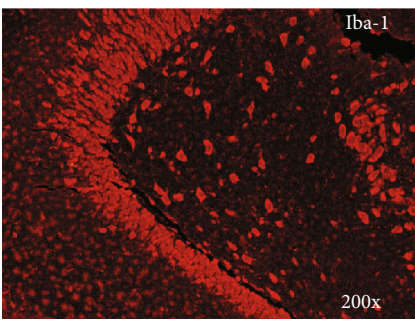

(d)

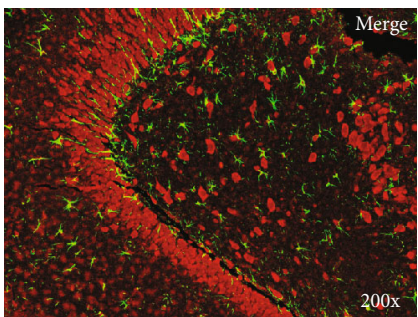

(f)

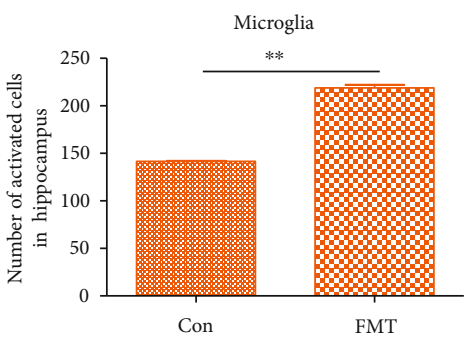

(h)

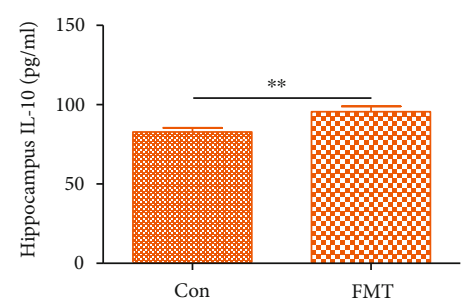

(j)

FIGURE 5: FMT modulates microglia, astrocyte, and inflammation activation in the hippocampus of the depression rat (200x, scale bar: $50 \mu \mathrm{m})$. GFAP and IBA-1 immunoreactivities are shown in the hippocampus of control (a, c, e) or FMT (b, d, f) rats. (g, h) Quantitative analysis of the number of activated astrocytes and microglia in each group. Normalization to the total cell number has been performed for quantification. (i) Proinflammatory cytokine IL-1 $\beta$ was increased after FMT. (j) Anti-inflammatory cytokine IL-10 was increased after FMT. All data are represented by the mean $\pm \operatorname{SEM}(n=6-7)$ by $t$-test; ${ }^{*} p<0.05,{ }^{* *} p<0.01$, and ${ }^{* * *} p<0.001$.

$[21,22]$, while Clostridium is usually associated with inflammation and contains several highly pathogenic species, including Clostridium botulinum and Clostridium tetani, which are known to produce toxins $[23,25,36]$. Hence, we proposed the ratio of $\mathrm{L} / \mathrm{C}$ as a potential index for the process of depression. Based on the alterations in the abovementioned gut microbiome, future studies should focus on the species of microbiome that changes significantly, as these species may serve as important targets for the treatment of depression. 
To investigate the pathological role of the gut microbiome in the development of depression, an FMT experiment was conducted [29]. We demonstrated that FMT in antibiotic-treated rats results in depression-like behavior, suggesting that the gut microbiome can act as a pathogen. In addition, the production of LPS by gramnegative bacteria can increase intestinal permeability and leaky gut in patients with depression [37]. In the present study, FABP2 and zonulin were increased after FMT, which are the major regulators of tight junctions between the endothelium and epithelium, regulating the intestinal and bloodbrain barriers [11]. FMT rats may have experienced altered intestinal permeability, exposing the liver to intestinal bacterial products and affecting liver metabolism.

Intestinal blood can be collected in the liver through the portal vein. Specific bacterial products such as lactic acid, organic acids, tryptophan, and propionic acid are related to animal behavior. Similarly, metabolites such as fatty acids, LPS, peptidoglycan, acylglycerol, sphingomyelin, and cholesterol can affect intestinal permeability to activate the gutbrain-liver-neural axis to regulate glucose homeostasis [38]. Moreover, metabolites produced by gut microbiota can be sensed by liver immune cells, which can disrupt liver homeostasis, leading to fibrosis and liver cancer [10]. In this research, we found that FMT changed liver metabolites markedly in antibiotic-treated rats. Compared with the control group rats, the levels of amino acids, including glutamate, glutamine, aspartate, serine, methionine, valine, leucine, lysine, and isoleucine, were significantly changed in FMT group rats. Previous studies have shown that amino acids play an important role in the brain. For example, glutamine is present in the brain as a neurotransmitter and promotes glutamatergic neurotransmission when it acts on astrocytes [39]. In the current study, valine and isoleucine levels were decreased in the liver of FMT rats. These results suggest that amino acid metabolism in FMT rats is disturbed. The liver is primarily responsible for the energy metabolism of the host. Glucose is an important supplier of host energy, and in our research, a significant reduction in glucose was observed in FMT rats compared to control rats, and this finding is consistent with previous research [30]. Similarly, lactic acid, which is involved in glycolysis and the tricarboxylic acid cycle, was also significantly downregulated. Taken together, the above results indicate that liver energy metabolism is disturbed by changes in the gut microbiome. Additionally, previous studies have found that glucose changes are also observed in serum and urine metabolomics studies in patients with depression [40]. An analysis of the fecal and serum metabolites of an FMT mouse model of depression has revealed that the level of carbohydrate metabolism in mice with depression increases [29]. A combination of previous research and the present study shows that depression-like microbiome leads to disorders of glucose metabolism in the liver. As the brain needs glucose to supply energy, disorders of liver glucose metabolism may lead to insufficient energy for the brain, which may promote the development of depression. The results of this study indicate that metabolic disorders are mainly focused on glycolysis and the tricarboxylic acid cycle, indicating that the pathological process of depression is accompanied by an energy metabolism disorder and that the liver may play a vital role.

Moreover, the pathogenesis of depression is related to disorders of oxidative stress [41]. In the current study, we found that the metabolite glutathione was significantly upregulated in FMT rats. Free radical scavenging in the brain is performed by glutathione, and glutathione deficiency impairs the antioxidant activity of the central nervous system [42]. In addition, oxidative damage caused by glutathione deficiency induces cell apoptosis [43]. In this experiment, increased liver glutathione levels helped to increase antioxidant activity, which may have protected FMT rats.

It has been reported that mice with depression develop lipid metabolism disorders [44]. In our research, lipidrelated metabolites were shown to characterize trends in the livers of FMT rats. There was a significant change in glycerol and arachidonic acid levels. Triglycerides are metabolized by the host to produce glycerol. Thus, these results indicate that lipid metabolism was disordered in FMT rats. In addition, disordered lipid metabolism is also found in fecal metabolites [45]. Metabolic disorders of major metabolites in the livers of depression patients may be the cause of high comorbidity between depression and metabolic syndromes [45].

Guida et al. have shown that nonneuronal cells (astrocytes and microglia) in the brain are involved in regulating synaptic plasticity in the cortical circuits involved in emotional processing [46]. In particular, increased proinflammatory factors and the activation of microglia in the brain play a crucial role in the development of depression [47]. An increase in the number of microglia and astrocytes indicates a shift in the brain to an inflammatory phenotype [48]. In our research, FMT caused the significant activation of astrocytes in the hippocampus, and proinflammatory cytokine IL- $1 \beta$ increased in the hippocampus of rats after FMT. IL-1 $\beta$ directly induces the proliferation of glia [49], which was also increased in FMT rats in this research. The expression of the anti-inflammatory cytokine IL-10 is increased in rats after FMT, which may be caused by IL- $1 \beta$, and plays an important role in protecting the brain. Researches have shown that the pathogenesis of depression is caused by a deficiency in 5-HT [34, 50]. Here, 5-HT expression was decreased in plasma in FMT rats. The intestinal barrier disruption we mentioned above may result in the inhibition of enterochromaffin cells (EC) and the inability to produce or release sufficient 5-HT.

There are some limitations in this experiment that need to be explained. First, the study was conducted only in male rats, and more clinical trials are needed to verify the results. Second, we only performed metabolomics analysis on the liver. The metabolomics analysis of the feces was not performed in the study, and the role of intestinal flora metabolites in our findings is still unclear.

In summary, our results indicate that depression can be transmitted through gut microbiome. More importantly, we proposed the ratio of $\mathrm{L} / \mathrm{C}$ as a potential index for the development of depression. The specific gut microbiome structure disrupts the integrity of the intestinal barrier, which in turn leads to disturbances in liver metabolism and inflammation in the brain. Here, we complement the previous evidence of 
the microbiome-gut-liver-brain axis and propose a novel index for depression. These findings provide a new perspective for the study of the pathogenesis of depression and provide potential targets for the development of drugs for depression.

\section{Materials and Methods}

4.1. Animal Treatment. Eight-week-old male SPF SD rats (10 weeks), each weighing $200 \pm 20 \mathrm{~g}$, were purchased from the Experimental Animal Center of Southern Medical University (Guangzhou, China). After one-week habituation, all rats were divided into the following two groups: control group (Con) and CUMS model group (Mod) and were housed under standard environmental conditions $\left(22 \pm 0.5^{\circ} \mathrm{C}, 50 \pm 5 \%\right.$ humidity, and a $12 \mathrm{~h}$ light $/ 12 \mathrm{~h}$ dark cycle) and maintained with free access to a standard laboratory pellet diet and water. A series of variable stimuli were performed on the model group (CUMS-treated) rats as previously described [51]. Rats were individually housed and repeatedly exposed to a set of CUMS stressors consisting of exposure to a set of CUMS stressors as follows: cage tilting for $24 \mathrm{~h}$, damp sawdust for $24 \mathrm{~h}(200 \mathrm{ml}$ water per individual cage, which is enough to make the sawdust bedding wet), noises for $1 \mathrm{~h}$ (alternative periods of $60 \mathrm{dBA}$ noise for $10 \mathrm{~min}$ and $10 \mathrm{~min}$ of silence), swimming in $4^{\circ} \mathrm{C}$ cold water for $5 \mathrm{~min}$, exposure to an experimental room at $50^{\circ} \mathrm{C}$ for $5 \mathrm{~min}, 24 \mathrm{~h}$ of food deprivation, $24 \mathrm{~h}$ of water deprivation, tail clamping for $1 \mathrm{~min}, 15$ shocks at unpredictable times ( $15 \mathrm{~mA}$, one shock $/ 5 \mathrm{~s}, 10 \mathrm{~s}$ duration), and restricted movement for $4 \mathrm{~h}$. One stressor was applied in random order each day, and the whole stress procedure lasted for 28 days in a completely random order. The study was approved by the Southern Medical University Experimental Animal Ethics Committee. All experimental procedures were performed in accordance with the relevant guidelines approved by the Experimental Animal Ethics Committee of Southern Medical University.

\subsection{Behavioral Testing}

4.2.1. Sucrose Preference Test (SPT). The open field test was performed on the 28th day as previously described [52]. After 24 hours of water ban, each rat was placed in a single cage and two bottles containing water and $1 \%$ sucrose solution were placed. The ratio of the consumption of the sucrose solution to the amount of total solution consumed in one hour represents a parameter of the pleasure behavior.

4.2.2. Open Field Test (OFT). The test was performed as previously described. Briefly, all rats were individually tested in a device consisting of a black square substrate $(50 * 50 \mathrm{~cm})$ and a black wall $(50 \mathrm{~cm})$ [53]. Rats were placed in the corners of the device, and after 1 minute of adaptation, the rats were free to move for 5 minutes using a video-computerized tracking system. The total activity time is used as an indicator of activity, and the time spent in the central area (36\% of surface area) is used as an indicator of depression behavior.
4.2.3. Tail Suspension Test (TST). The rat tail was hung on an iron frame (head $20 \mathrm{~cm}$ from the ground). The duration of immobility in the rats was monitored by a time recorder over 4 minutes. When they did not show any body movements, were on passive suspension, and had no movement at all, the mice were considered to be stationary [54].

4.3. Sample Collection. Anesthesia was administered with sodium pentobarbital. Blood was collected and centrifuged at $3000 \mathrm{rpm}$ for 5 minutes at $4^{\circ} \mathrm{C}$. Feces and the liver were collected, frozen in liquid nitrogen, and maintained at $-80^{\circ} \mathrm{C}$ for detection.

4.4. $16 S$ rRNA Gene Sequence Analysis. Total genomic DNA of fecal samples was extracted by the InviMag stool DNA kit (Invitek, Germany). Fecal microbial DNA was extracted using Fast DNA SPIN extraction kits (MP Biomedicals, Santa Ana, CA, USA) and applied to amplification of the V3-V4 region of $16 \mathrm{~S}$ rDNA.

4.5. Fecal Microbiome Transplant Experiment. As described previously $[55,56]$, fecal stools collected randomly from 10 model and control group rats were used to colonize to antibiotic-treated rats. Briefly, fecal samples were suspended in PBS ( $15 \mathrm{ml} / \mathrm{g}$ stool), mixed in equal volumes, and then, these stool samples were vortexed for 5 minutes and centrifuged $(1000 \mathrm{rpm})$ for 5 minutes to precipitate fecal pellets. Antibiotic-treated rats were orally administered fecal suspension $200 \mu \mathrm{l}$ for three days. The behavior test was applied at day 7 after fecal microbiome transplant. To prepare the pseudo-germ-free model, rats were provided with ampicillin ( $1 \mathrm{~g} / \mathrm{l})$, kanamycin $(5 \mathrm{~g} / \mathrm{l})$, vancomycin $(500 \mathrm{mg} / \mathrm{l})$, neomycin trisulfate $(1 \mathrm{~g} / \mathrm{l})$, and metronidazole $(1 \mathrm{~g} / \mathrm{l})$ in drinking water for three weeks. All antibiotics were purchased from SigmaAldrich.

4.6. Liquid Chromatography-Mass Spectrometry. LC-MS preparation was performed as previously described [29]. Liver tissue samples $(60 \mathrm{mg}$ ) were homogenized with $200 \mu \mathrm{l}$ of ultrapure water; then, $800 \mu \mathrm{l}$ of methanol/acetonitrile $(1: 1, v / v)$ was added for vortexing and sonication for $30 \mathrm{~min}$. Samples were separated by UHPLC and subjected to mass spectrometry using a Triple TOF 5600 mass spectrometer (AB SCIEX).

4.7. Quantitative Image Analysis. Immunofluorescence was performed as previously described [57], with the following modification: primary antibody: rabbit anti-GFAP (1:2000; Ab5076/Ab10062, Abcam, UK) overnight at $4^{\circ} \mathrm{C}$. For detection of primary antibodies, a suitable secondary antibody conjugated to FITC-conjugated donkey anti-mouse IgG (1:400, A21202, Life Technologies, USA) was used. The sample was covered with a mounting medium (S2100, Solarbio, China) and examined with an epifluorescence microscope (Nikon Eclipse 80i, Nikon, Japan).

4.8. Pro-/Anti-inflammatory Cytokines and Markers of Intestinal Barrier Analysis. Proinflammatory cytokine IL$1 \beta$, anti-inflammatory cytokine IL-10, production of enterochromaffin cell 5-HT, and markers of gut dysbiosis 
and gut permeability zonulin and FABP2 in the hippocampus and/or in the plasma were tested using an ELISA kit (Cusabio, Houston, TX, USA; https://www.cusabio.com/).

4.9. Statistical Analysis. Statistical analyses were approached using SPSS version 22 (SPSS, Inc., Chicago, IL, USA) and GraphPad Prism 5. The results such as $\alpha$-diversity, behavior data, body weight, IL-1 $\beta$, IL-10, 5 -HT, zonulin, and FABP2 were presented as the mean \pm SEM.

Differences between two groups were assessed using unpaired two-tailed Student's $t$-test.

\section{Abbreviations}

5-HT: Serotonin

CUMS: Chronic unpredictable mild stress

FMT: $\quad$ Fecal microbiota transplant

IF: $\quad$ Immunofluorescence

EC: $\quad$ Enterochromaffin cells

ELISA: Enzyme-linked immunosorbent assay

BW: $\quad$ Body weight

SPT: $\quad$ Sucrose preference test

OFT: $\quad$ Open field test

TST: $\quad$ Tail suspension test

PCA: $\quad$ Principal component analysis

PCoA: $\quad$ Principal coordinates analysis

PLS-DA: Partial least squares discrimination analysis

F/B: $\quad$ Firmicutes to Bacteroidetes

L/C: $\quad$ Lactobacillus to Clostridium

FABP2: $\quad$ Fatty acid binding protein-2

LPS: Lipopolysaccharide

OPLS-DA: Orthogonal PLS-DA

LC-MS: Liquid chromatograph mass spectrometer

FDR: $\quad$ False discovery rate

PC: $\quad$ Phosphatidylcholine

GFAP: Glial fibrillary acidic protein

Iba-1: $\quad$ Ionized calcium binding adaptor molecule-1

IL-1 $\beta$ : Interleukin 1

IL-10: Interleukin 10

SPF: $\quad$ Specific pathogen free

OTU: Operational taxonomic unit

IgG: Immunoglobulin G

UHPLC: Ultra-high-performance liquid chromatography

FITC: $\quad$ Fluorescein isothiocyanate isomer I.

\section{Data Availability}

All data needed to evaluate the conclusions in the paper are present in the paper and/or Supplementary Materials. Additional data related to this paper may be requested from the authors.

\section{Conflicts of Interest}

The authors declare no conflict of interest.

\section{Authors' Contributions}

Wei-jie Lv and Xiao-ling Wu designed the work, performed the research study, and drafted the manuscript. Wen-qian
Chen, Yue-fei Li, and Gui-feng Zhang participated in experimental work. Ao Guo, Jia-hao Zhou, and Li-min Chao analyzed the database. Cui Liu and Shi-ning Guo designed and supervised the research study. All authors read and approved the final manuscript. Wei-jie Lv and Xiao-ling Wu contributed equally.

\section{Acknowledgments}

This work was supported by the National Natural Science Foundation of China (No. 31472232) and the Department of Agriculture of Guangdong Province, the Project of SCAU TCVM Training Base.

\section{Supplementary Materials}

Fig. S1: alterations of microbiota after CUMS. Fig. S2: taxon abundance changes in phylum and genus levels. Fig. S3: orthogonal partial least squares discrimination analysis (OPLS-DA) score. Fig. S4: metabolite hierarchical clustering. Fig. S5: construction of the aminoacyl-tRNA biosynthesis metabolism pathway in rats. Fig. S6: change of 5HT in plasma. Table S1: metabolites identified in liver extracts. Table S2: changed pathways with $p<0.05$ and Supplementary Methods. (Supplementary Materials)

\section{References}

[1] G. E. Simon, "Social and economic burden of mood disorders," Biological Psychiatry, vol. 54, no. 3, pp. 208-215, 2003.

[2] E. H. Reynolds, "Brain and mind: a challenge for WHO," Lancet, vol. 361, no. 9373, pp. 1924-1925, 2003.

[3] J. P. Guilloux, G. Douillard-Guilloux, R. Kota et al., "Molecular evidence for BDNF- and GABA-related dysfunctions in the amygdala of female subjects with major depression," Molecular Psychiatry, vol. 17, no. 11, pp. 1130-1142, 2012.

[4] L. Sun, L. Ma, H. Zhang et al., "Fto deficiency reduces anxietyand depression-like behaviors in mice via alterations in gut microbiota," Theranostics, vol. 9, no. 3, pp. 721-733, 2019.

[5] A. Naseribafrouei, K. Hestad, E. Avershina et al., "Correlation between the human fecal microbiota and depression," Neurogastroenterology and Motility, vol. 26, no. 8, pp. 11551162, 2014.

[6] Z. X. Zhao, J. Fu, S. R. Ma et al., "Gut-brain axis metabolic pathway regulates antidepressant efficacy of albiflorin," Theranostics, vol. 8, no. 21, pp. 5945-5959, 2018.

[7] G. A. W. Rook, C. L. Raison, and C. A. Lowry, "Microbial 'old friends', immunoregulation and socioeconomic status," Clinical and Experimental Immunology, vol. 177, no. 1, pp. 1-12, 2014.

[8] A. E. Hoban, R. M. Stilling, F. J. Ryan et al., "Regulation of prefrontal cortex myelination by the microbiota," Translational Psychiatry, vol. 6, no. 4, p. e774, 2016.

[9] P. Zheng, Y. Wang, L. Chen et al., "Identification and validation of urinary metabolite biomarkers for major depressive disorder," Molecular \& Cellular Proteomics, vol. 12, no. 1, pp. 207-214, 2013.

[10] A. J. Macpherson, M. Heikenwalder, and S. C. Ganal-Vonarburg, "The liver at the nexus of host-microbial interactions," Cell Host \& Microbe, vol. 20, no. 5, pp. 561-571, 2016. 
[11] B. R. Stevens, R. Goel, K. Seungbum et al., "Increased human intestinal barrier permeability plasma biomarkers zonulin and FABP2 correlated with plasma LPS and altered gut microbiome in anxiety or depression," Gut, vol. 67, no. 8, pp. 15551557, 2018.

[12] A. J. Bruce-Keller, J. M. Salbaum, M. Luo et al., "Reply to: High-fat diet-induced dysbiosis as a cause of neuroinflammation," Biological Psychiatry, vol. 80, no. 1, pp. E5-E6, 2016.

[13] C. Yang, J. Gao, J. Zhang, and A. L. Luo, "Enterochromaffin cells in the gut: a distant regulator of brain function?," Gut, vol. 67 , no. 8, pp. 1557-1558, 2018.

[14] P. Willner, R. Muscat, and M. Papp, "Chronic mild stressinduced anhedonia: a realistic animal model of depression," Neuroscience and Biobehavioral Reviews, vol. 16, no. 4, pp. 525-534, 1992.

[15] S. C. Stanford, "The open field test: reinventing the wheel," Journal of Psychopharmacology, vol. 21, no. 2, pp. 134-135, 2007.

[16] L. Steru, R. Chermat, B. Thierry, and P. Simon, "The tail suspension test: a new method for screening antidepressants in mice," Psychopharmacology, vol. 85, no. 3, pp. 367-370, 1985.

[17] J. Sun, F. Wang, X. Hu et al., "Clostridium butyricum attenuates chronic unpredictable mild stress-induced depressivelike behavior in mice via the gut-brain axis," Journal of Agricultural and Food Chemistry, vol. 66, no. 31, pp. 8415-8421, 2018.

[18] A. Burokas, S. Arboleya, R. D. Moloney et al., "Targeting the microbiota-gut-brain axis: prebiotics have anxiolytic and antidepressant-like effects and reverse the impact of chronic stress in mice," Biological Psychiatry, vol. 82, no. 7, pp. 472487, 2017.

[19] M. L. Wong, A. Inserra, M. D. Lewis et al., "Inflammasome signaling affects anxiety- and depressive-like behavior and gut microbiome composition," Molecular Psychiatry, vol. 21, no. 6, pp. 797-805, 2016.

[20] J. Foster and K. A. Neufeld, "Gut-brain axis: how the microbiome influences anxiety and depression," International Journal of Neuropsychopharmacology, vol. 17, pp. 27-27, 2014.

[21] J. Lee, W. Yang, A. Hostetler et al., "Characterization of the anti-inflammatory Lactobacillus reuteri BM36301 and its probiotic benefits on aged mice," BMC Microbiology, vol. 16, no. 1, 2016.

[22] H. Li, L. Zhang, L. Chen, Q. Zhu, W. Wang, and J. Qiao, "Lactobacillus acidophilus alleviates the inflammatory response to enterotoxigenic Escherichia coli K88 via inhibition of the NF- $\kappa \mathrm{B}$ and p38 mitogen-activated protein kinase signaling pathways in piglets," BMC Microbiology, vol. 16, no. 1, p. 273, 2016.

[23] H. Yu, K. Chen, Y. Sun et al., "Cytokines are markers of the Clostridium difficile-induced inflammatory response and predict disease severity," Clinical and Vaccine Immunology, vol. 24 , no. 8, 2017.

[24] J. Limsrivilai, K. Rao, R. W. Stidham et al., "The systemic inflammatory response to Clostridium difficile infection (Cdi) in patients with ulcerative colitis," Gastroenterology, vol. 152, no. 5, pp. S760-S760, 2017.

[25] R. D. Heijtz, S. Wang, F. Anuar et al., "Normal gut microbiota modulates brain development and behavior," Proceedings of the National Academy of Sciences of the United States of America, vol. 108, no. 7, pp. 3047-3052, 2011.
[26] J. Henao-Mejia, E. Elinav, C. Jin et al., "Inflammasome-mediated dysbiosis regulates progression of NAFLD and obesity," Nature, vol. 482, no. 7384, pp. 179-185, 2012.

[27] S. R. Konstantinov and M. P. Peppelenbosch, "Fecal microbiota transfer may increase irritable bowel syndrome and inflammatory bowel diseases-associated bacteria," Gastroenterology, vol. 144, no. 4, pp. E19-E20, 2013.

[28] V. K. Ridaura, J. J. Faith, F. E. Rey et al., "Gut microbiota from twins discordant for obesity modulate metabolism in mice," Science, vol. 341, no. 6150, article 1241214, 2013.

[29] P. Zheng, B. Zeng, C. Zhou et al., "Gut microbiome remodeling induces depressive-like behaviors through a pathway mediated by the host's metabolism," Molecular Psychiatry, vol. 21, no. 6, pp. 786-796, 2016.

[30] B. Li, K. Guo, L. Zeng et al., "Metabolite identification in fecal microbiota transplantation mouse livers and combined proteomics with chronic unpredictive mild stress mouse livers," Translational Psychiatry, vol. 8, no. 1, p. 34, 2018.

[31] P. F. Devaux, "Static and dynamic lipid asymmetry in cell membranes," Biochemistry, vol. 30, no. 5, pp. 1163-1173, 1991.

[32] J. Pearson-Leary, C. Zhao, K. Bittinger et al., "The gut microbiome regulates the increases in depressive-type behaviors and in inflammatory processes in the ventral hippocampus of stress vulnerable rats," Molecular Psychiatry, 2019.

[33] B. C. Hains and S. G. Waxman, "Activated microglia contribute to the maintenance of chronic pain after spinal cord injury," Journal of Neuroscience, vol. 26, no. 16, pp. 43084317, 2006.

[34] I. Mahar, F. R. Bambico, N. Mechawar, and J. N. Nobrega, "Stress, serotonin, and hippocampal neurogenesis in relation to depression and antidepressant effects," Neuroscience and Biobehavioral Reviews, vol. 38, pp. 173-192, 2014.

[35] H. Jiang, Z. Ling, Y. Zhang et al., "Altered fecal microbiota composition in patients with major depressive disorder," Brain, Behavior, and Immunity, vol. 48, pp. 186-194, 2015.

[36] J. Maukonen, R. Satokari, J. Matto, H. Soderlund, T. Mattila-Sandholm, and M. Saarela, "Prevalence and temporal stability of selected clostridial groups in irritable bowel syndrome in relation to predominant faecal bacteria," Journal of Medical Microbiology, vol. 55, no. 5, pp. 625-633, 2006.

[37] J. K. Kiecolt-Glaser, H. M. Derry, and C. P. Fagundes, "Inflammation: depression fans the flames and feasts on the heat," The American Journal of Psychiatry, vol. 172, no. 11, pp. 1075-1091, 2015.

[38] C. Cabou, P. D. Cani, G. Campistron et al., "Central insulin regulates heart rate and arterial blood flow: an endothelial nitric oxide synthase-dependent mechanism altered during diabetes," Diabetes, vol. 56, no. 12, pp. 2872-2877, 2007.

[39] T. Eid, N. Tu, T. S. W. Lee, and J. C. K. Lai, "Regulation of astrocyte glutamine synthetase in epilepsy," Neurochemistry International, vol. 63, no. 7, pp. 670-681, 2013.

[40] P. Zheng, H. C. Gao, Q. Li et al., "Plasma metabonomics as a novel diagnostic approach for major depressive disorder," Journal of Proteome Research, vol. 11, no. 3, pp. 1741-1748, 2012.

[41] T. M. Michel, D. Pulschen, and J. Thome, "The role of oxidative stress in depressive disorders," Current Pharmaceutical Design, vol. 18, no. 36, pp. 5890-5899, 2012. 
[42] J. W. Gawryluk, J. F. Wang, A. C. Andreazza, L. Shao, L. N. Yatham, and L. T. Young, "Prefrontal cortex glutathione Stransferase levels in patients with bipolar disorder, major depression and schizophrenia," The International Journal of Neuropsychopharmacology, vol. 14, no. 08, pp. 1069-1074, 2011.

[43] J. B. Schulz, J. Lindenau, J. Seyfried, and J. Dichgans, "Glutathione, oxidative stress and neurodegeneration," European Journal of Biochemistry, vol. 267, no. 16, pp. 4904-4911, 2000.

[44] G. Chen, D. Yang, Y. Yang et al., "Amino acid metabolic dysfunction revealed in the prefrontal cortex of a rat model of depression," Behavioural Brain Research, vol. 278, pp. 286292, 2015.

[45] V. Vaccarino, C. McClure, B. D. Johnson et al., "Depression, the metabolic syndrome and cardiovascular risk," Psychosomatic Medicine, vol. 70, no. 1, pp. 40-48, 2008.

[46] F. Guida, F. Turco, M. Iannotta et al., "Antibiotic-induced microbiota perturbation causes gut endocannabinoidome changes, hippocampal neuroglial reorganization and depression in mice," Brain, Behavior, and Immunity, vol. 67, pp. 230-245, 2018.

[47] D. Rial, C. Lemos, H. Pinheiro et al., "Depression as a glialbased synaptic dysfunction," Frontiers in Cellular Neuroscience, vol. 9, 2016.

[48] D. M. Norden, P. J. Trojanowski, E. Villanueva, E. Navarro, and J. P. Godbout, "Sequential activation of microglia and astrocyte cytokine expression precedes increased Iba-1 or GFAP immunoreactivity following systemic immune challenge," Glia, vol. 64, no. 2, pp. 300-316, 2016.

[49] J. Pearson-Leary, D. Eacret, R. Chen, H. Takano, B. Nicholas, and S. Bhatnagar, "Inflammation and vascular remodeling in the ventral hippocampus contributes to vulnerability to stress," Translational Psychiatry, vol. 7, no. 6, p. e1160, 2017.

[50] K. G. Margolis, K. Stevanovic, Z. Li et al., "Pharmacological reduction of mucosal but not neuronal serotonin opposes inflammation in mouse intestine," Gut, vol. 63, no. 6, pp. 928-937, 2014.

[51] X. Gao, X. Zheng, Z. Li et al., "Metabonomic study on chronic unpredictable mild stress and intervention effects of Xiaoyaosan in rats using gas chromatography coupled with mass spectrometry," Journal of Ethnopharmacology, vol. 137, no. 1, pp. 690-699, 2011.

[52] S. Pothion, J. C. Bizot, F. Trovero, and C. Belzung, "Strain differences in sucrose preference and in the consequences of unpredictable chronic mild stress," Behavioural Brain Research, vol. 155, no. 1, pp. 135-146, 2004.

[53] C. S. Kim, P. Y. Chang, and D. Johnston, "Enhancement of dorsal hippocampal activity by knockdown of HCN1 channels leads to anxiolytic- and antidepressant-like behaviors," Neuron, vol. 75, no. 3, pp. 503-516, 2012.

[54] R. Crupi, M. Cambiaghi, L. Spatz et al., "Reduced adult neurogenesis and altered emotional behaviors in autoimmune-prone B-cell activating factor transgenic mice," Biological Psychiatry, vol. 67, no. 6, pp. 558-566, 2010.

[55] K. Mao, A. P. Baptista, S. Tamoutounour et al., "Innate and adaptive lymphocytes sequentially shape the gut microbiota and lipid metabolism," Nature, vol. 554, no. 7691, pp. 255259, 2018.
[56] O. Koren, J. K. Goodrich, T. C. Cullender et al., "Host remodeling of the gut microbiome and metabolic changes during pregnancy," Cell, vol. 150, no. 3, pp. 470-480, 2012.

[57] M. F. Sun, Y. L. Zhu, Z. L. Zhou et al., "Neuroprotective effects of fecal microbiota transplantation on MPTP-induced Parkinson's disease mice: Gut microbiota, glial reaction and TLR4/TNF- $\alpha$ signaling pathway," Brain, Behavior, and Immunity, vol. 70, pp. 48-60, 2018. 


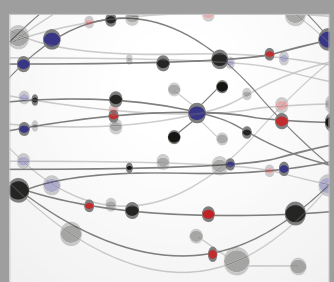

The Scientific World Journal
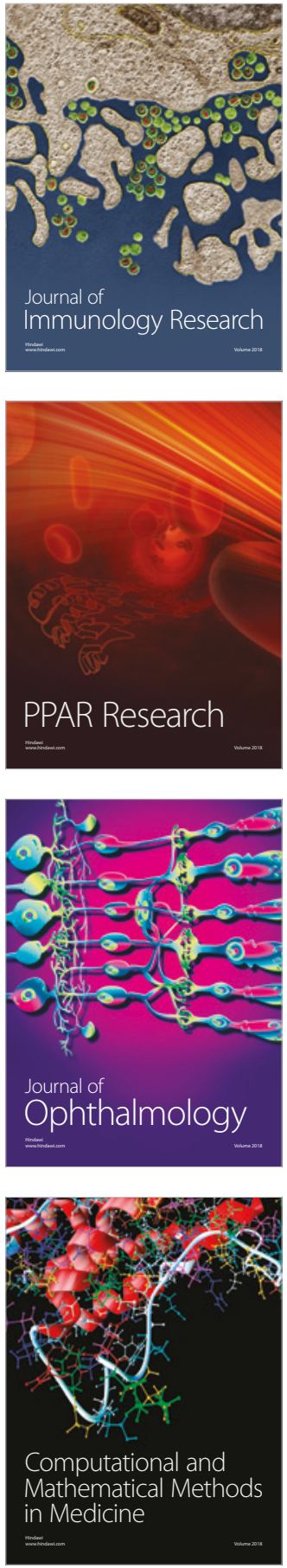

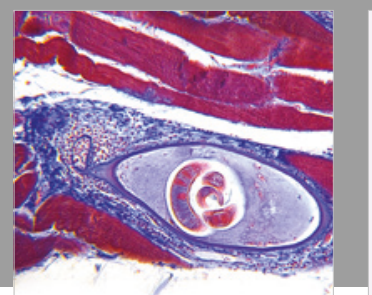

Gastroenterology Research and Practice

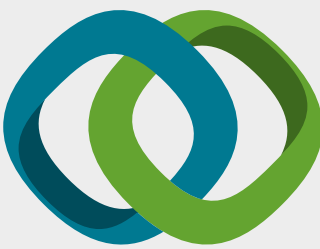

\section{Hindawi}

Submit your manuscripts at

www.hindawi.com
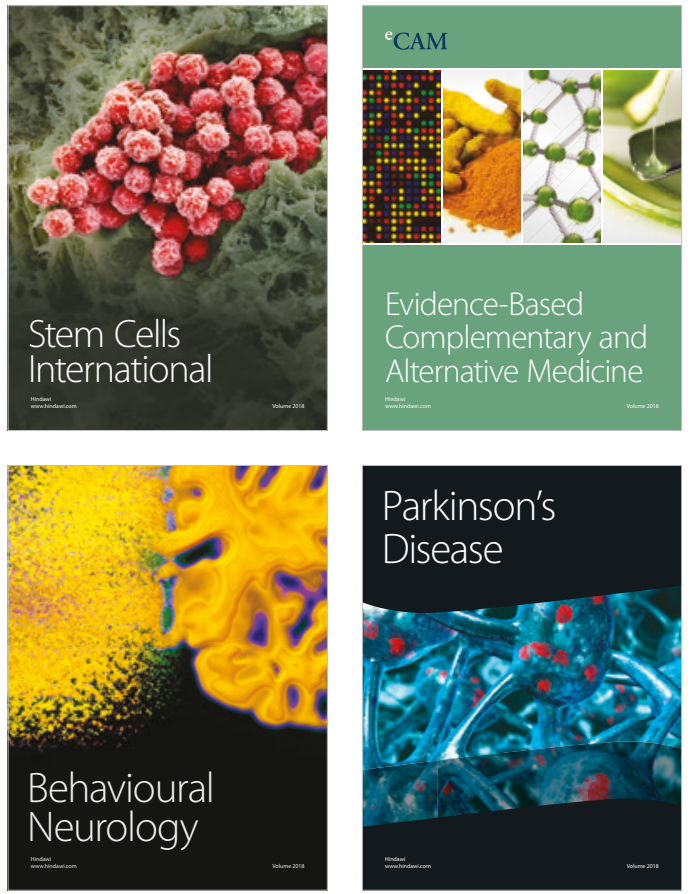

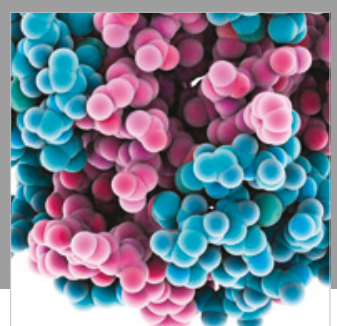

ournal of

Diabetes Research

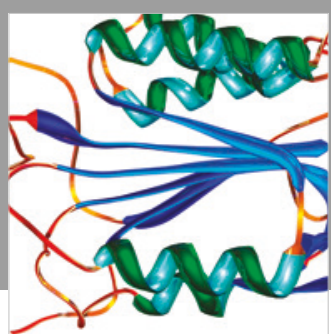

Disease Markers
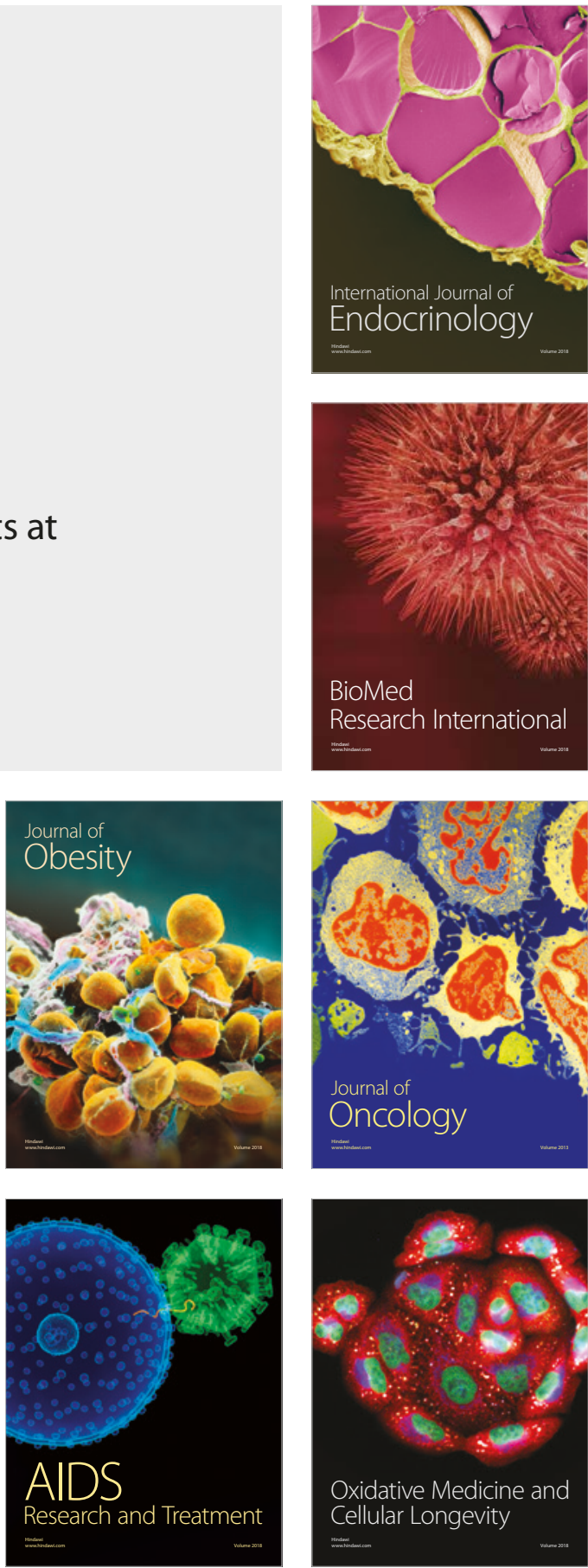